\title{
Cartographie automatique des caractéristiques pluviométriques: Prise en compte des relations pluviométrie-morphométrie
}

\author{
Automatic mapping of rainfall data: \\ Study of rainfall - Morphometric relationships
}

\author{
J.-P. Laborde \\ École Nationale Supérieure de Géologie Appliquée \\ et de Prospection Minière \\ Institut National Polytechnique de Lorraine
}

\section{Préambule}

L'étude que nous présentons a été réalisée par le Laboratoire de Mécanique et d'Hydraulique de l'Ecole Nationale Supérieure de Géologie Appliquée de Nancy, à la demande et avec le concours des Services Régionaux d'Aménagement des Eaux d'Alsace et de Lorraine et de l'Agence Financière de Bassin Rhin-Meuse, dans le cadre de la Mission Déléguée de Bassin.

\section{Introduction}

Ces dernières années ont vu le développement et la mise en oeuvre pratique de nouvelles méthodes d'estimation des débits de crue :

- la méthode du "gradex" pour les crues de fréquence très rare ;

- la méthode "SOCOSE" du Ministère de l'Agriculture pour les crues des petits bassins versants ;

- la méthode "CRUPEDIX";

- les nouvelles notes techniques pour le dimensionnement des ouvrages en assainissements urbains ;

- etc.

Ces estimations des débits sont basées sur la connaissance de différents paramètres caractéristiques des pluies extrêmes. Il était souhaitable de foumir aux projeteurs une étude synthétique des pluies sur la région Nord-Est de la France, en prenant en compte le maximum de l'information disponible.

Cela était possible du fait de l'existence d'une masse suffisante de données pluviométriques et du développement d'une bibliothèque de traitements statistiques et cartographiques à l'ENSG. Cette bibliothèque CARTOLAB est conçue, à l'origine, pour la résolution de problèmes d'estimation minière, mais ces derniers présen- tent des analogies très étroites avec le problème climatologique envisagé. Les objectifs de cette étude étaient donc doubles, à la fois régionaux et méthodologiques.

Nous tenons à remercier ici ceux sans qui ce travail n'aurait pu se faire : en premier lieu, les différents observateurs qui ont une tâche essentielle mais obscure, et le personnel du Bureau de l'Eau de la Météorologie Nationale qui a assumé la charge ingrate de la critique de ces données.

Enfin, nous ne donnerons ici qu'une présentation succincte, mais l'on pourra se référer à l'étude SRAEL citée en bibliographie pour y trouver toute information plus détaillée.

\section{Caractéristiques pluviométriques retenues}

\section{Les données pluviométriques de base}

Les informations pluviométriques sont stockées à l'Agence Financière de Bassin sur support informatique. Les observations émanent de plusieurs services : Météorologie Nationale bien-sûr, mais aussi SRAE, DDA, CNRF, INRA, etc.

Cette banque de données est à l'origine, hétérogène quant à sa qualité. Cependant, nous avons eu la chance de voir ces données critiquées par le Bureau de l'Eau de la Météorologie Nationale. En particulier, et ce qui était essentiel pour l'étude des pluies extrêmes, il ne subsistait plus de cumulés non signalés dans le fichier critiqué.

L'information pluviométrique de départ consistait donc en observations sur 364 postes, et pour une période critiquée allant de 1949 à 1976 .

Des Etudes précédentes, portant sur les pluies journalières extrêmes [3], ont montré que le régime pluviométrique de la région opposait deux saisons : un 
"hiver" de novembre à avril, et un "été", de mai à octobre. Nous souhaitions, par ailleurs, établir les paramètres des distributions statistiques des pluies extrêmes sur une période commune d'observations. Le choix de cette période devait répondre à deux impératifs contradictoires : une durée suffisamment longue pour que la précision des estimations statistiques soit satisfaisante, et une durée suffisamment courte pour que

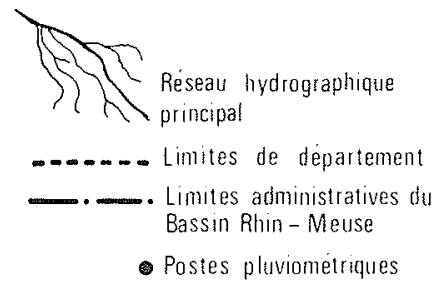

Fìgure 1 - Localisation générale de la zone étudiée.

\section{Les gradex saisonniers}

Pour chaque station, nous avons pu vérifier le comportement asymptotiquement exponentiel des lois de distribution des pluies extrêmes. Ce comportement est mis en évidence par le report dans un graphique de Gumbel (fig. 2) des pluies journalières maximales mensuelles de chaque "saison". On constate souvent que l'ajustement à une loi de Gumbel, figuré par un trait fin, n'est pas parfait. Nous avons préféré estimer le gradex par la pente de la droite figurée en trait fort qui est ajustée au mieux dans le nuage des pluies de fréquence supérieure à 0.5 , (soit d'après 60 pluies maximales mensuelles).

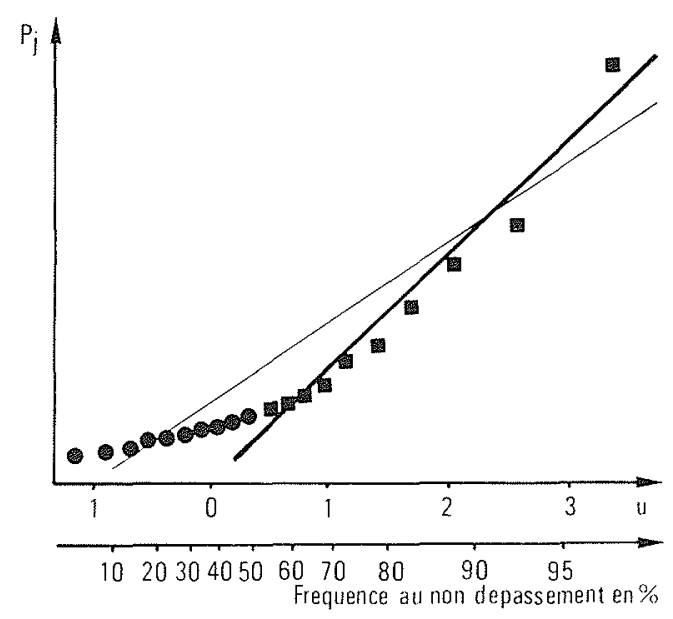

Figure 2 - Schéma des distributions expérimentales dans un graphique de GUMBEL. le nombre de stations disponibles permette une synthèse cartographique. Notre choix s'est porté sur la période de vingt ans $(1957$ - 1976), où nous disposons de 102 postes pluviométriques.

Comme le montre la figure 1, ces stations sont assez bien réparties dans l'espace, et une période de vingt ans permet une étude statistique significative.

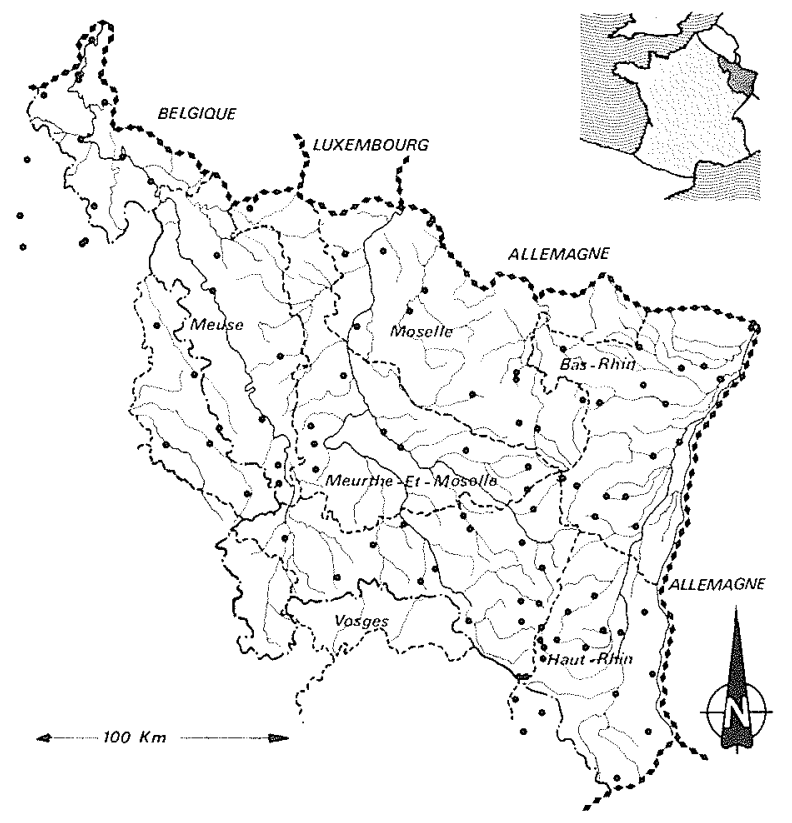

Cette estimation des gradex est bien sûr entachée d'une erreur d'échantillonnage. Une étude expérimentale basée sur cinq stations longues de la région, montre que l'on peut estimer l'incertitude sur les gradex à environ $\pm 20 \%$.

\section{Les pluies journalières décennales (Fig. 3)}

Les pluies journalières décennales sont estimées par composition des lois de distribution des pluies jour-

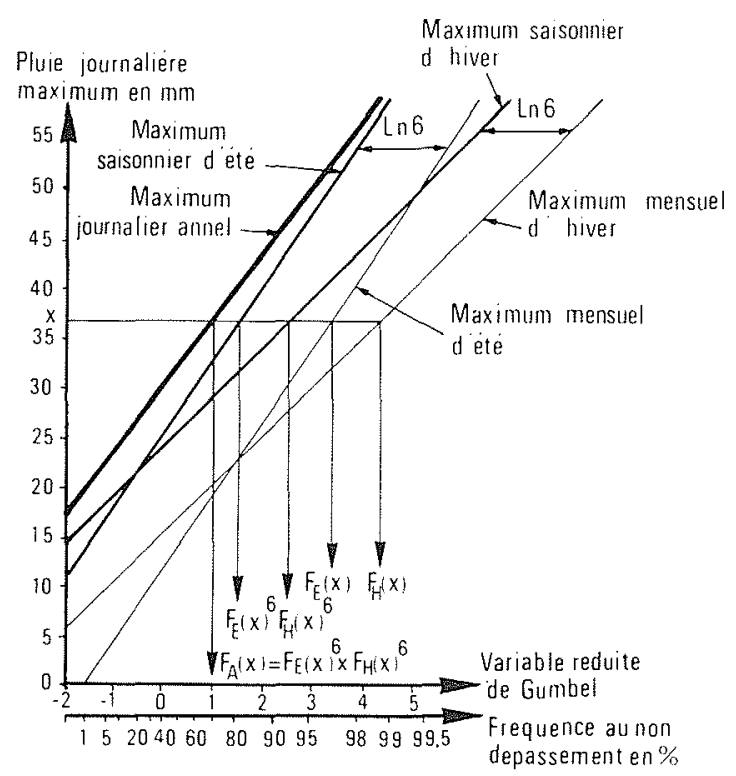

Figure 3 - Estimation de la loi de distribution des pluies maximales annuelles par composition des lois de distributions saisonnières et mensuelles. 
nalières maximales mensuelles de chaque saison. La probabilité pour qu'une hauteur de pluie soit non dépassée durant toute l'année est sensiblement égale au produit des probabilités pour qu'elle soit non dépassée chacun des six mois de l'hiver et des six mois de l'été. A partir des lois de distribution des pluies maximales mensuelles d'hiver et d'été on passe aux pluies maximales saisonnières d'hiver et d'été. Enfin, en composant ces deux lois, on obtient les pluies maximales annuelles. On peut estimer que les pluies journalières décennales sont connues à $\pm 10 \%$, compte tenu des vingt années d'observation.

\section{Statistiques ponctuelles aux différents postes pluvio- métriques}

Le tableau I récapitule l'ensemble des résultats obtenus pour chaque station.
Les pluies maximales mensuelles d'hiver $\mathrm{X}$ exprimées en $1 / 10^{\text {ème }}$ de $\mathrm{mm}$. ont pour loi de distribution :

$$
\operatorname{Prob}(X \leqslant x)_{H}=F_{H}(x)=e^{-e^{-\frac{x-X_{O H}}{G_{H}}}}
$$

De même pour les pluies d'été :

$$
\operatorname{Prob}(X \leqslant x)_{E}=F_{E}(x)=e^{-e^{-\frac{x-X_{O E}}{G_{E}}}}
$$

avec

$$
\begin{aligned}
& X_{O H}=\text { mode d'hiver } \\
& X_{O E}=\text { mode d'été } \\
& G_{H}=\text { gradex d'hiver } \\
& G_{E}=\text { gradex d'été. }
\end{aligned}
$$

\begin{tabular}{|c|c|c|c|c|c|c|c|c|c|c|c|}
\hline Nom des sta & $\mathrm{GH}$ & $\mathrm{O}_{\mathrm{H}}$ & CII & $O_{E}$ & $\mathrm{PIO}$ & Non des st & $\mathrm{GH}$ & $0_{1}$ & $\mathrm{GE}$ & $o_{1}$ & 10 \\
\hline 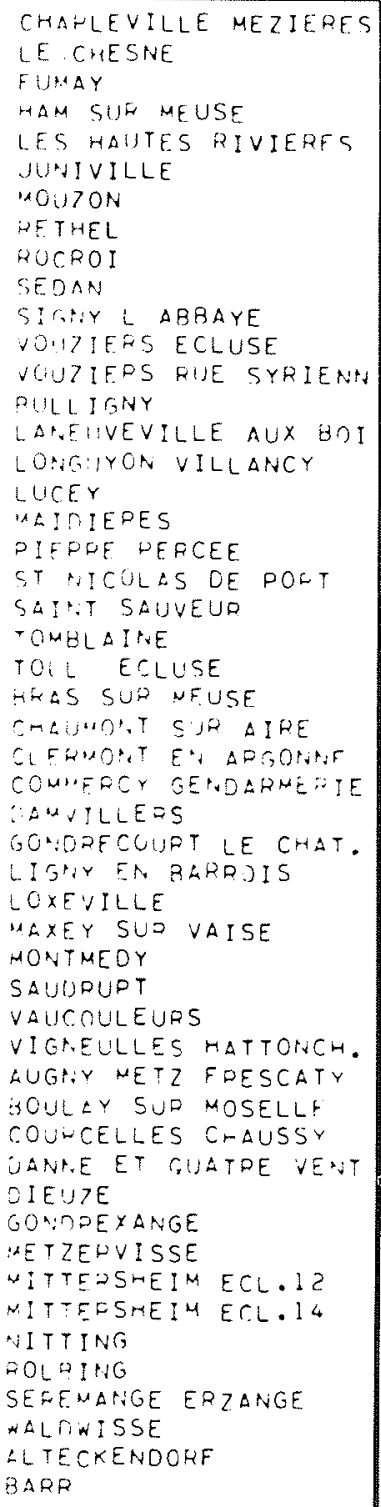 & \begin{tabular}{|l|}
56 \\
57 \\
79 \\
82 \\
66 \\
56 \\
58 \\
49 \\
106 \\
51 \\
57 \\
50 \\
58 \\
61 \\
78 \\
468 \\
71 \\
66 \\
91 \\
73 \\
74 \\
46 \\
54 \\
63 \\
73 \\
72 \\
78 \\
52 \\
73 \\
156 \\
70 \\
92 \\
90 \\
74 \\
75 \\
55 \\
59 \\
54 \\
45 \\
58 \\
67 \\
75 \\
44 \\
62 \\
52 \\
78 \\
69 \\
50 \\
55 \\
56 \\
73
\end{tabular} & 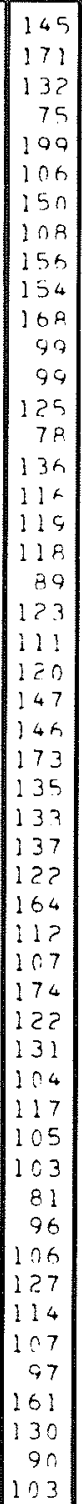 & 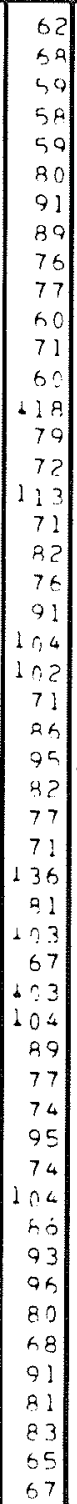 & $\begin{array}{l}152 \\
151 \\
160 \\
145 \\
190 \\
129 \\
110 \\
111 \\
142 \\
131 \\
185 \\
141 \\
152 \\
110 \\
125 \\
173 \\
127 \\
164 \\
192 \\
151 \\
152 \\
118 \\
131 \\
131 \\
157 \\
149 \\
172 \\
125 \\
137 \\
173 \\
78 \\
159 \\
139 \\
154 \\
134 \\
130 \\
118 \\
136 \\
129 \\
127 \\
131 \\
103 \\
128 \\
105 \\
141 \\
148 \\
148 \\
129 \\
133 \\
146 \\
163 \\
136\end{array}$ & $\begin{array}{l}430 \\
454 \\
478 \\
441 \\
493 \\
461 \\
498 \\
474 \\
612 \\
455 \\
455 \\
442 \\
412 \\
540 \\
479 \\
491 \\
542 \\
473 \\
567 \\
481 \\
541 \\
539 \\
545 \\
472 \\
528 \\
578 \\
515 \\
470 \\
497 \\
677 \\
521 \\
543 \\
504 \\
579 \\
567 \\
486 \\
459 \\
440 \\
512 \\
444 \\
531 \\
531 \\
442 \\
537 \\
484 \\
473 \\
511 \\
470 \\
488 \\
434 \\
451\end{array}$ & 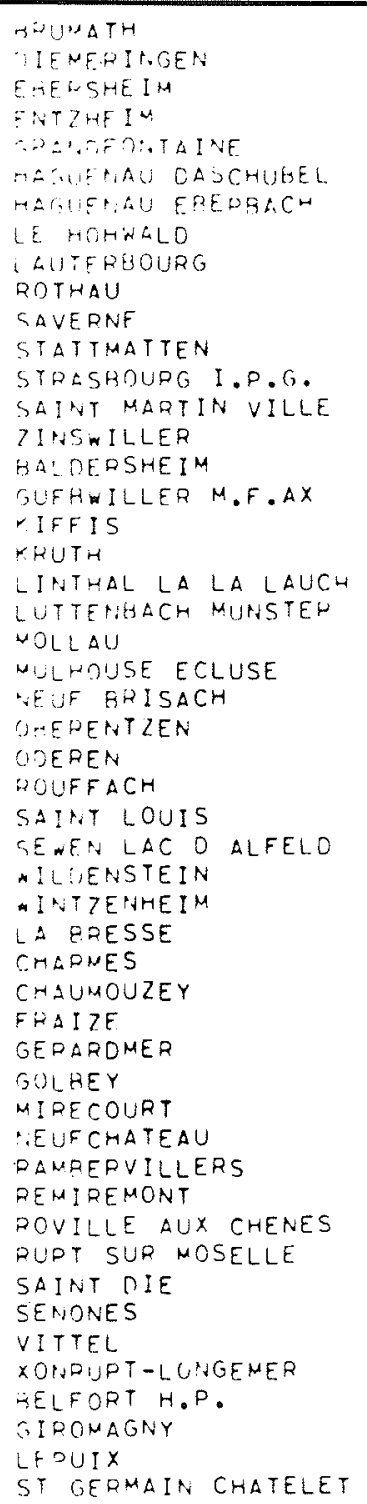 & 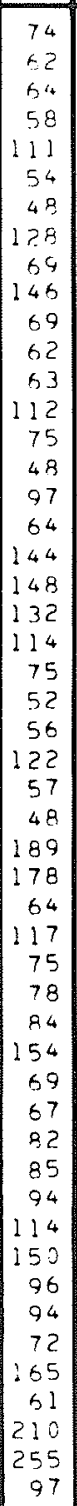 & 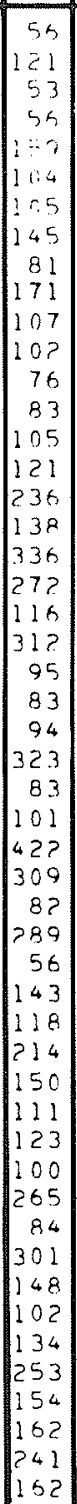 & $\begin{array}{r}95 \\
93 \\
49 \\
87 \\
105 \\
106 \\
111 \\
107 \\
86 \\
97 \\
65 \\
108 \\
84 \\
97 \\
55 \\
89 \\
83 \\
91 \\
109 \\
109 \\
85 \\
113 \\
92 \\
96 \\
89 \\
107 \\
107 \\
66 \\
147 \\
94 \\
142 \\
79 \\
58 \\
85 \\
112 \\
109 \\
105 \\
130 \\
172 \\
80\end{array}$ & 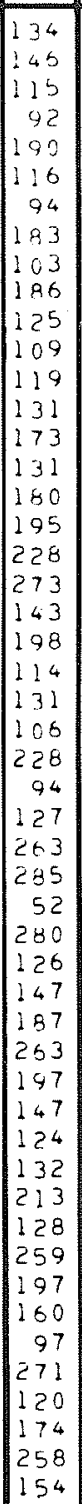 & $\begin{array}{l}528 \\
494 \\
411 \\
449 \\
560 \\
547 \\
544 \\
722 \\
470 \\
781 \\
433 \\
550 \\
469 \\
541 \\
472 \\
493 \\
649 \\
569 \\
932 \\
498 \\
667 \\
807 \\
509 \\
320 \\
468 \\
841 \\
529 \\
401 \\
1204 \\
1032 \\
628 \\
775 \\
406 \\
532 \\
576 \\
049 \\
495 \\
443 \\
569 \\
523 \\
690 \\
048 \\
924 \\
572 \\
555 \\
566 \\
940 \\
553 \\
1029 \\
1307 \\
584\end{array}$ \\
\hline
\end{tabular}

Enfin, la dernière colonne, notée $\mathrm{P}_{10}$, donne l'estimation de la pluie journalière décennale, (hauteur de 
pluie dépassée en moyenne une fois tous les dix ans), et exprimée en $1 / 10^{\text {èmes }}$ de $\mathrm{mm}$.

\section{Caractéristiques morphométriques}

\section{Constitution d'une banque de données altimétriques}

La zone à cartographier s'inscrit dans un rectangle orienté Nord-Sud, d'environ 200 sur $300 \mathrm{~km}$. Nous avons décidé d'introduire la topographie sous forme numérique, en échantillonnant les altitudes aux noeuds d'un réseau à mailles carrées. La dimension des mailles devait être suffisamment fine pour amener une bonne précision du tracé à l'échelle envisagée $\left(1 / 500000^{e ̀ m e}\right)$, tout en étant assez grande pour que le nombre de mailles couvrant la zone permette un traitement aisé et rapide sur l'ordinateur utilisé (CII Iris 80).

Pour ces raisons, le fichier a été constitué à partir des cartes topographiques au $1 / 100000^{\mathrm{èm}} \mathrm{e}$. Nous y avons relevé les altitudes aux noeuds d'un réseau à mailles carrées de $2,5 \mathrm{~km}$ de côté. Compte tenu des dimensions de la zone à cartographier, c'est plus de 12000 altitudes qui ont été échantillonnées. Le bloc-diagramme de la figure 4 illustre la représentativité de ce fichier, puisque nous y retrouvons tous les éléments caractéristiques du relief, en particulier les côtes de Meuse et de Moselle.

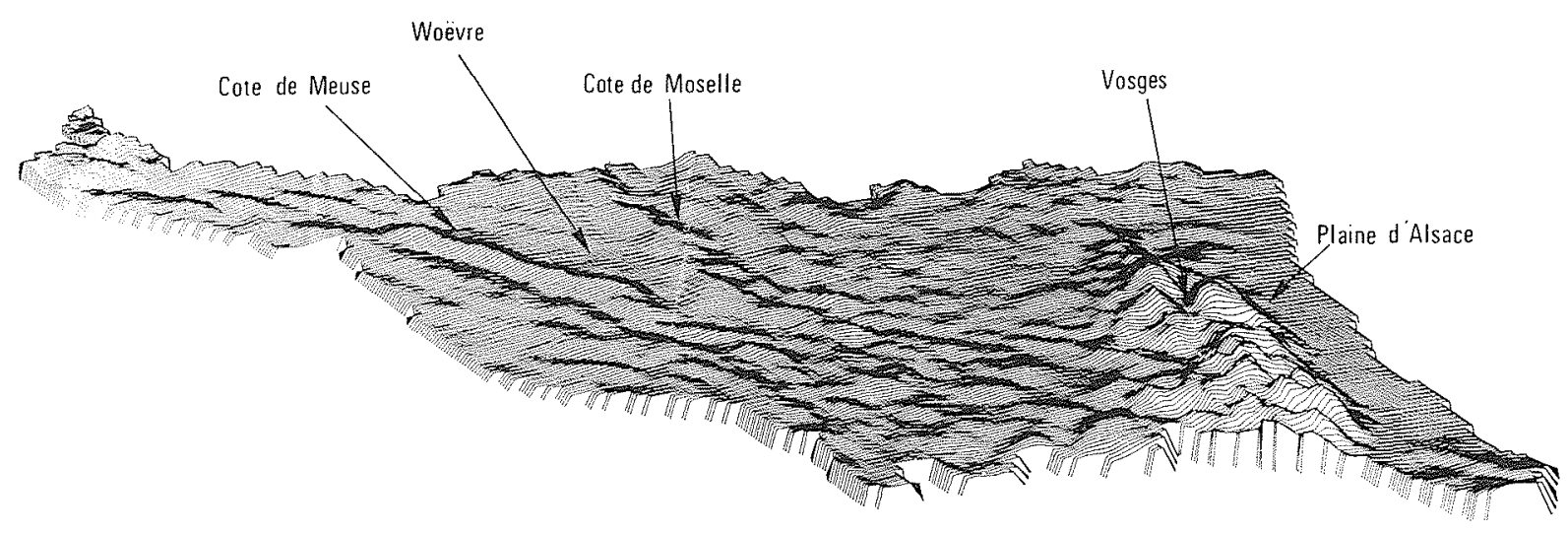

Figure 4 - Bloc-diagramme du Nord-Est de la France.

\section{Paramètres altimétriques}

Il est connu de longue date, que l'altitude joue un rôle très important sur la pluviométrie. Mais de quelles altitudes s'agit-il ? Altitude du pluviomètre ? Altitude des sommets environnants? ...

Nous avons donc envisagé cinq définitions :

- l'altitude réelle $\mathrm{Z}$ du poste pluviométrique ;

- l'altitude ZFXY : altitude du poste pluviométrique interpolée par une fonction $Z(x, y)$ de degré 4 en $x$ et en $y$ et calée sur les 25 noeuds du réseau les plus proches; - 1'altitude ZFXYL : approximation de Lagrange, basée sur les 25 noeuds les plus proches (lissage); - l'altitude ZFXYLL : approximation de Lagrange, basée sur les 64 noeuds les plus proches (lissage plus intense) ;

- l'altitude ZFS : ce mode de lissage était déjà utilisé manuellement ; son but est de ne retenir dans la topographie, que les reliefs susceptibles d'affecter les mouvements des masses d'air.

Des études précédentes [10] ont montré que seules, dans la région, les vallées dont la largeur dépasse $4 \mathrm{~km}$ environ, affectent la répartition des pluies.

Pour calculer la cote ZFS d'un point M (fig. 5), on se déplace au nœud du réseau le plus proche et on retient la valeur maximale parmi la cote réelle à ce noeud et les quatre cotes obtenues par interpolation linéaire à partir des huit autres noeuds les plus proches.

Comme le suggère la figure 6 (à 2 dimensions), on "efface" ainsi les vallées dont la largeur est inférieure à 2 mailles, soit $5 . \mathrm{km}$.

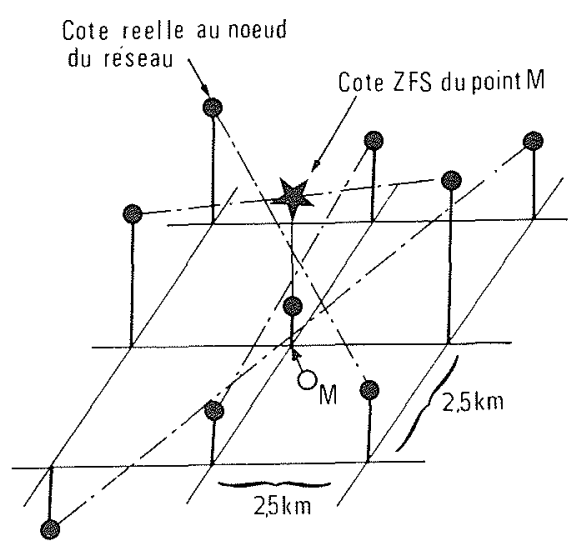

Figure 5 - Estimation de la cote ZFS.

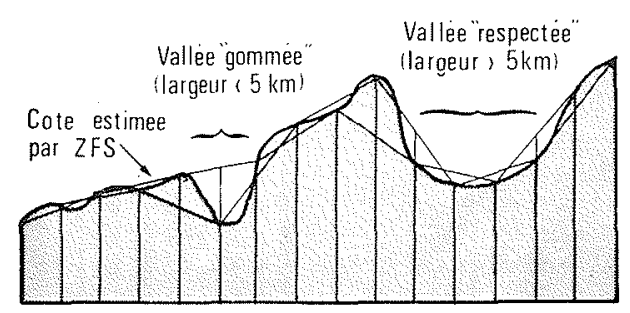

Figure 6 - Effet de l'emploi de ZFS pour des vallées d'amplitude supérieure ou inférieure à $5 \mathrm{~km}$. 


\section{Caractérisation de l'encaissemenl d'un sile (fig. 7)}

L'altitude n'est pas le seul paramètre topographique qui caractérise un site. Supposons que nous soyons en $M$ à l'abri d'une crête montagneuse. L'influence de cette crête est d'autant plus forte qu'elle est élevée par rapport à $M(d z)$ et qu'elle en est proche (dl). Pour tenir compte à la fois des aspects, dénivelée et distance, il est logique de faire intervenir la tangente de l'angle $\theta$ $(\tan \theta=\mathrm{dz} / \mathrm{dl})$ sous lequel on voit la crête depuis $M$ et selon la direction des vents dominants. Par ailleurs, $\tan \theta$ ne peut à elle-seule, mesurer l'influence de la crête ; à cela deux raisons; d'une part, un pic isolé aura moins d'influence qu'une crête continue ; d'autre part, les vents s'orientent dans une certaine "fourchette" d'azimuts autour de la direction principale.

Nous proposons donc de caractériser l'encaissement d'un site à partir de l'angle solide sous lequel on voit l'horizon entre deux azimuts limites Az.1 et Az.2.

Dans notre région, les perturbations d'hiver viennent généralement de l'Ouest ou du Sud-Ouest. En été par contre, il semble que ce soit plutôt l'encaissement (creux ou bosses) qui affecte la répartition des pluies d'orage.

Sur cette idée, nous avons construit six paramètres :

- AS1 : rapport de l'angle solide sous lequel on voit 1'horizon entre les azimuts 0 et 360 degrés à l'angle théorique maximal. Cet angle est calculé uniquement sur un diamètre de $20 \mathrm{~km}$ entourant le point considéré ;

- AS2 : même définition que AS1, mais pour des azimuts allant de 180 à 360 degrés (vision vers l'Ouest) ; AS3 : même définition que AS1, mais pour des azimuts de 180 à 270 degrés (vision vers le Sud-Ouest) ;

Ces trois paramètres varient de -1 à +1 . Ils sont négatifs sur un sommet et positifs dans un creux.

TG1 : tangente moyenne sous laquelle on voit 1'horizon, estimée à partir des 25 noeuds les plus proches et entre les azimuts 0 et 360 degrés;

- TG2 : même définition, mais pour des azimuts de 180 à 360 degrés ;

- TG3 : même définition, mais pour des azimuts de 180 à 270 degrés ;

Ces trois demiers paramètres varient dans le même sens que les $A S$, mais de $-\infty+\infty$.

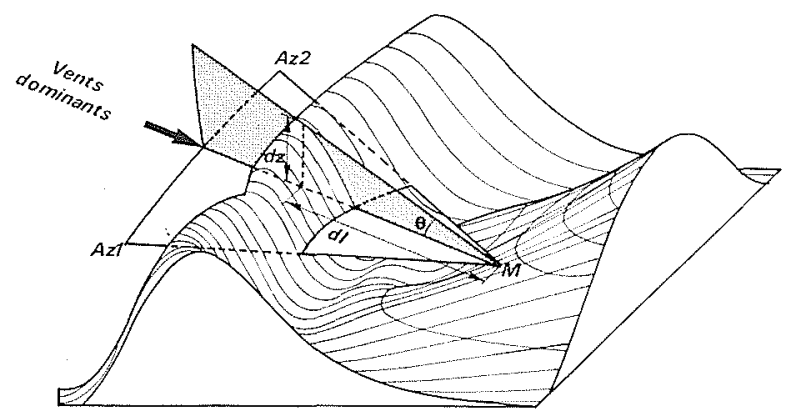

Figure 7 - Caractérisation d'un site par l'angle solide sous lequel on voit l'horizon.

\section{Etude des relations pluviométrie-morphométrie}

A ce stade, nous disposions donc, d'une part, de caractéristiques ponctuelles de pluies, et d'autre part, des valeurs ponctuelles des paramètres morphométriques pouvant influer sur la pluviométrie. Soit $P(x, y)$, la valeur de la pluie en un point $\mathrm{M}$; nous avons postulé que $P(x, y)$ dépendait d'une première composante $f_{S}(x, y$, $z)$ fonction qui caractérise la topographie sur le domaine $D$ entourant le poste ; $f_{S}(x, y, z)$ est en quelque sorte un effet de site qui doit intégrer l'altitude et l'orientation des versants. Nous supposerons que cette fonction $f_{S}(x, y, z)$ est la même en tout point de la région.

Il existe certainement une deuxième fonction $R(x, y)$ qui traduit la répartition régionale des pluies corrigées des effets de site. Par ailleurs, il se peut très bien que la fonction $f_{s}(x, y, z)$ change à l'intérieur du domaine d'étude : par exemple, le gradient altimétrique des pluies n'est pas forcément exactement le même sur le versant lorrain que sur le versan alsacien des Vosges. Cependant, les erreurs qui en découlent sont vraisemblablement analogues sur toute une zone, et elles seront, en partie, prises en compte dans la fonction $R(x, y)$.

Nous avons donc certainement une relation du type :

$$
P(x, y)=f_{S}(x, y, z)+R(x, y)
$$

Dans un premier temps, nous avons essayé d'évaluer la fonction $f_{S}(x, y, z)$ à partir des différentes altitudes représentatives du site, et des différentes tangentes moyennes sous lesquelles on voit l'horizon. Le choix du nombre et des paramètres à utiliser a été guidé par l'étude des régressions multiples linéaires.

Pour les pluies journalières décennales $\mathrm{P} 10$ et les gradex d'hiver GH, seule l'altitude ZFS joue un rôle statistiquement significatif :

$$
P 10=0.0534 \mathrm{ZFS}+35.5+\epsilon_{P 10}
$$

avec $P 10$ en $\mathrm{mm} / \mathrm{j}$ et $\mathrm{ZFS}$ en $\mathrm{m}$, soit un gradient altimétrique de $5 \mathrm{~mm} / 100 \mathrm{~m}$ (le coefficient de corréaltion est alors de 0.85 );

$$
G H=0.116 \mathrm{ZFS}+34.3+\epsilon_{G H}
$$

avec $G H$ en $1 / 10 \mathrm{~mm} / \mathrm{j}$ et $\mathrm{ZFS}$ en $\mathrm{m}$, soit un gradient altimétrique de $1 \mathrm{~mm} / 100 \mathrm{~m}$ (le coefficient de corrélation est alors de 0.82).

Les gradex d'été GE s'expliquent moins bien par la topographie, mais on y voit néanmoins l'influence statistiquement significative de l'altitude ZFS et aussi de l'encaissement TG :

$$
G E=0.0402 \mathrm{ZFS}-16.0 \mathrm{TG}+74.9+\epsilon_{G E}
$$

avec $G E$ en $1 / 10 \mathrm{~mm} / \mathrm{j} ;$ ZFS en $\mathrm{m}$ et TG sans unité (le coefficient de corrélation multiple est 0.46 ).

Le gradient altimétrique de $0.4 \mathrm{~mm} / 100 \mathrm{~m}$ est beaucoup plus faible que pour GH ; d'autant plus qu'en moyenne, lorsque ZFS augmente, TG augmente également. Il faut noter ici que le coefficient affecté à TG est négatif, ce qui signifie qu'à altitude égale, les gradex d'été sont plus forts sur les bosses que dans les creux. Il faut $y$ voir certainement une localisation préférentielle des orages d'été sur les sommets. 


\section{Cartographie automatique}

La cartographie automatique s'effectue en trois phases:

- calculs des résidus $\epsilon$ à chaque poste pluviométrique ; - interpolation de ces $\epsilon$ aux noeuds d'un maillage régulier (différents a priori du maillage de $2.5 \mathrm{~km} \times 2.5$ $\mathrm{km}$ et en général plus lâche), et cartographie des $\epsilon$; - cartographie finale à partir de la carte des résidus $\epsilon$ et des données morphométriques.

\section{Cartographie des résidus}

Les résidus ayant été calculés sur les 102 stations pluviométriques, il convient d'interpoler leur valeur aux noeuds d'un réseau régulier. Parmi plusieurs techniques utilisables, nous avons choisi celle des filtres autorégressifs (analogue au krigeage). Dans cette méthode, les points d'échantillonnage sont rapportés, dans un premier temps, aux noeuds les plus proches du réseau. $\mathrm{Si}$ plusieurs points sont ramenés au même noeud, la valeur affectée au noeud en sera la moyenne arithmétique. On conçoit donc que l'on obtienne un lissage de plus en plus intense en diminant le maillage de la zone étudiée. On effectue alors plusieurs essais en augmentant le nombre de mailles jusqu'à atteindre la précision souhaitée.

Dans notre cas, nous avons retenu une précision telle que dans $70 \%$ des cas, l'écart entre la valeur réelle et celle cartographiée soit inférieur au quart de l'incertitude sur les estimations ponctuelles de départ.

Les trois cartes ainsi obtenues présentent une organisation régionale nette, avec des zones synclinales et anticlinales bien localisées, comme le montre la figure 8 .

\section{Cartographie finale}

Nous disposons donc d'une carte de résidus la plus "lisse" possible (pour notre méthode d'interpolation

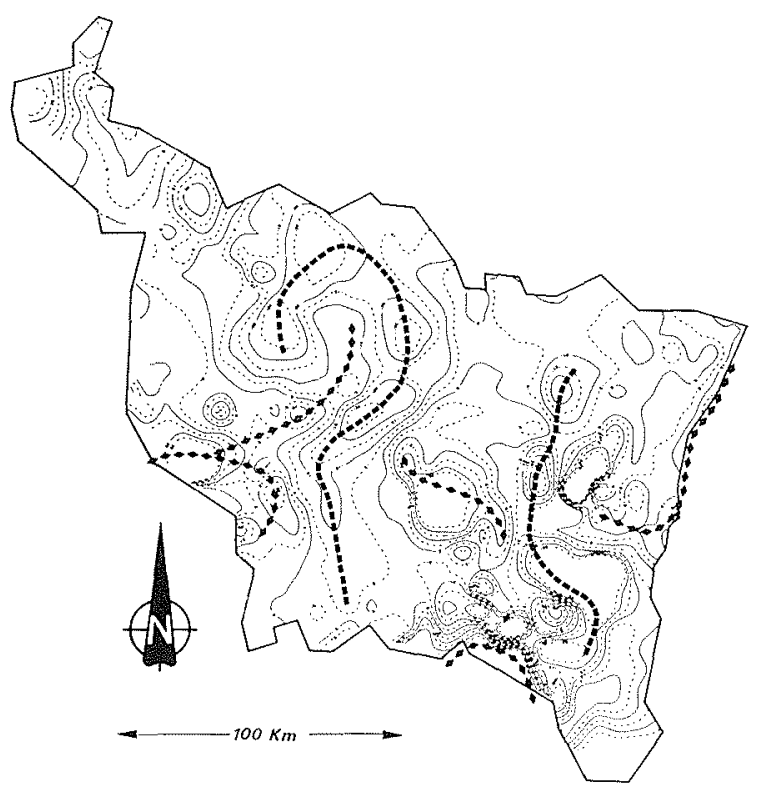

Figure 8 - Résidus des gradex des pluies journalières maximales mensuelles d'hiver (en $1 / 10 \mathrm{~mm} / \mathrm{j}$ ). et non pas au sens de fonction spline), compte tenu de la précision des données de départ, et aussi des fichiers morphométriques. En tout point du maillage primitif de $2.5 \mathrm{~km} \times 2.5 \mathrm{~km}$, nous pouvons estimer le paramètre à cartographier en prenant la valeur de $\epsilon$ cartée, et en y ajoutant l'infuence du site d'après les équations de régressions citées page 335 .

On constate que les courbes isohyètes de la figure 9 dessinent des détails justifiés par la connaissance que nous avons de la topographie. Cependant, les variations régionales sous-jacentes de la figure 8 sont les plus "lisses" possibles, car elles ne s'appuient que sur un nombre restreint de points de mesures (102) et sur des données entachées d'erreurs d'échantillonnage.

L'étude statistique des écarts entre les valeurs de GE, $\mathrm{GH}$ et P10 estimées aux postes pluviométriques et les valeurs cartographiées, montre que dans $70 \%$ des cas, l'erreur relative est inférieure à $\pm 6 \%$ pour les gradex d'hiver, à $\pm 5 \%$ pour les gradex d'été, et à $\pm 3.5 \%$ pour les pluies journalières décennales.

Les cartes 8 et 9 sont volontairement simplifiées ici. En effet, les versions originales comportent tous les renseignements ponctuels [15].

\section{Retour sur l'utilisation des paramètres morpho- métriques}

Arrivé au terme de ce travail, les enseignements sont de deux ordres. Ils concernent d'une part, la répartition des pluies extrêmes dans la France du Nord-Est, et d'autre part, la mise en évidence quantitative de l'influence du relief sur la pluviométrie.

Du point de vue des relations entre pluviométrie et morphométrie, on obtient une vision plus claire en procédant par analyse en composantes principales. Les quatorze variables que nous avons étudiée peuvent se ramener à trois composantes qui expliquent dans l'ordre $61 \%, 24 \%$ et $6 \%$ de la variance totale (les autres composantes tombent en-dessous de $3 \%$ ).

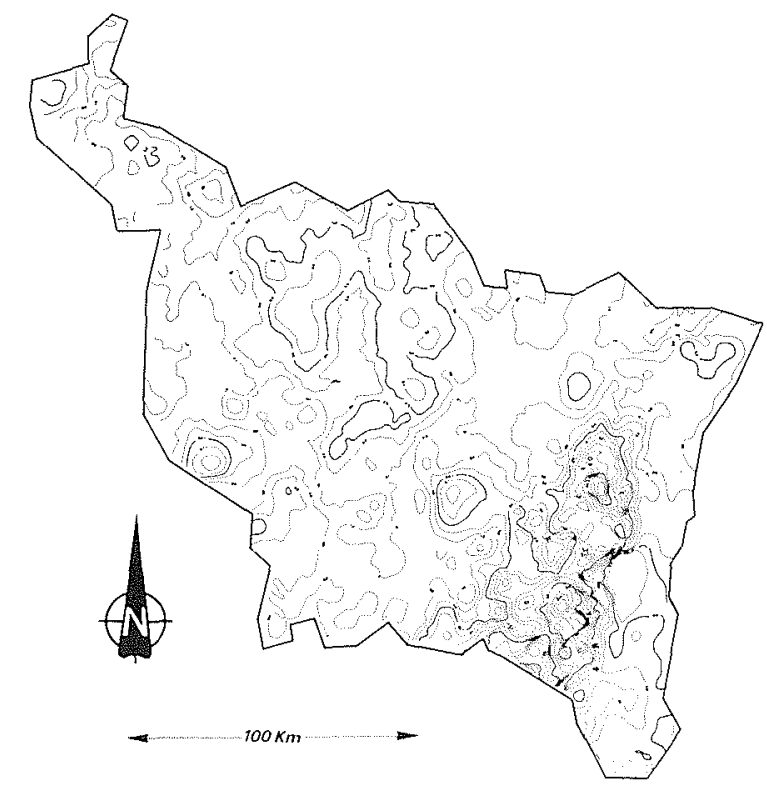

Figure 9 - Gradex des pluies journalières maximales mensuelles d'hiver (en $1 / 10 \mathrm{~mm} / \mathrm{j}$ ). 
La figure 10 représente la projection des quatorze variables dans le plan des deux premières composantes. Les caractéristiques morphométriques se regroupent d'une façon compacte autour de trois pôles:

- l'altitude du poste avec la variable $Z$ et l'estimation ZFXY de $Z$ à partir de l'échantillonnage d'altitudes ; - l'altitude moyenne autour du poste avec les variables ZFXYL, ZFXYLL et ZFS ;

- l'encaissement du site avec les six variables $A S$ et $T G$.

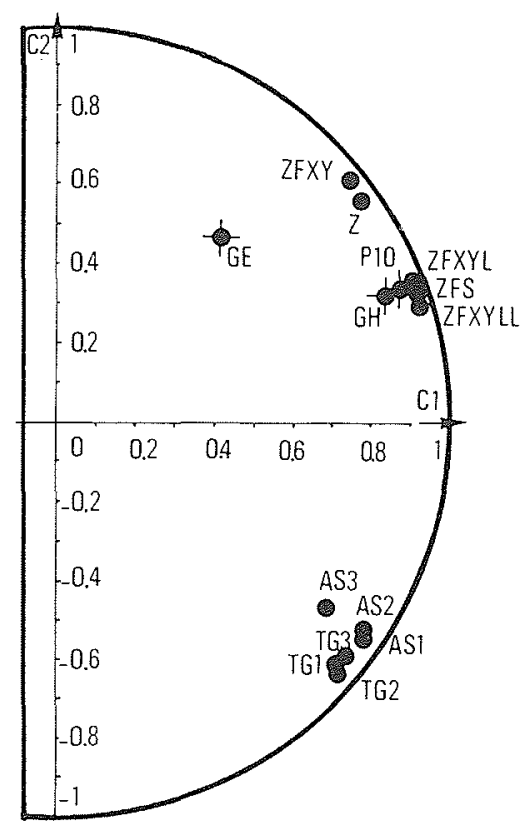

Figure 10 - Projection des variables dans le plan des deux premières composantes principales.

(Cette représentation est faite en tenant compte des trois variables pluviométriques, mais l'A.C.P., sur les seules variables topographiques, donne des résultats analogues).

La pluie décennale et le gradex des pluies d'hiver sont très proches du pôle altitude moyenne qui est essentiellement lié à la première composante. Le gradex des pluies d'été dépend à la fois de la première composante et de la deuxième, mais essentiellement de la troisième comme le montre la figure 11 .

Cette composante est pratiquement indépendante des paramètres topographiques.

Tableau 11 - Projection des variables sur les trois premières composantes

\begin{tabular}{|c|c|c|c|r|r|r|c|}
\hline & P10 & $G E$ & $G H$ & $Z$ & ZFXY & ZFXYL & ZFXYLL \\
\hline 1 & .866 & .413 & .831 & .771 & .741 & .906 & .932 \\
2 & .325 & .466 & .317 & .565 & .612 & .350 & .296 \\
3 & .318 & .741 & .131 & -.181 & -.216 & -.185 & -.170 \\
\hline
\end{tabular}

\begin{tabular}{|r|r|r|r|r|r|r|r|}
\hline & ZFS & AS1 & AS2 & AS3 & TG1 & TG2 & TG3 \\
\hline 1 & .924 & .781 & .783 & .692 & .710 & .715 & .731 \\
2 & .317 & -.557 & -.536 & -.473 & -.626 & -.640 & -.588 \\
3 & -.160 & -.065 & -.017 & -.049 & .068 & .109 & .078 \\
\hline
\end{tabular}

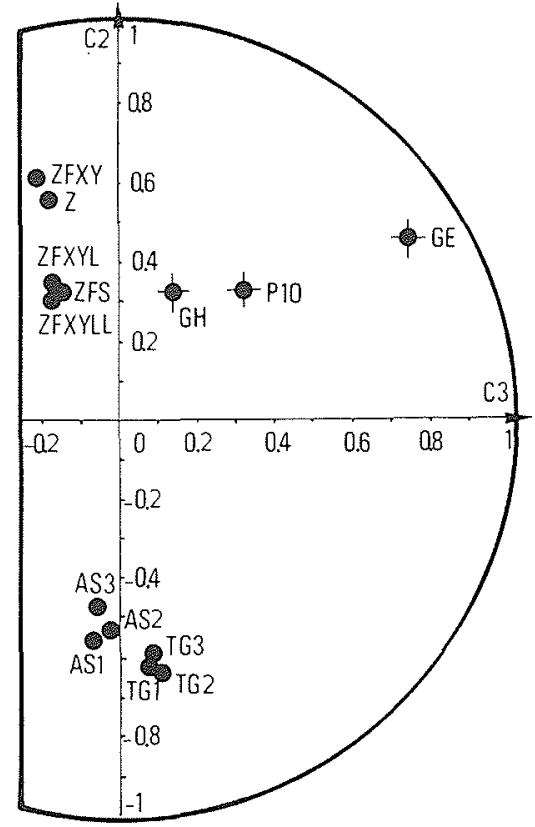

Figure 11 - Projection des variables dans le plan des deuxièmes et troisièmes composantes.

On peut expliquer a posteriori, les résultats obtenus par régressions multiples: $\mathrm{P} 10$ et $\mathrm{GH}$ sont très proches du pôle altitude moyenne.

Pour le gradex des pluies d'été GE, il dépend de la première composante représentant surtout les altitudes moyennes (première variable explicative dans les régressions multiples), mais aussi de la seconde que l'on peut choisir de représenter, soit par les angles solides, soit par les altitudes réelles.

La figure 12 présente la projection des variables dans le plan des deux premières composantes, calculées uniquement d'après les variables topographiques. Par une

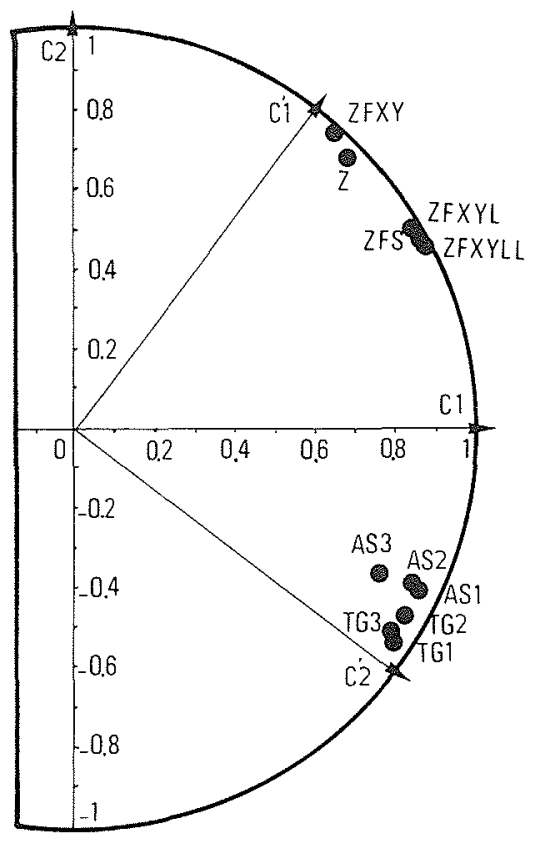

Figure 12 - Projection des variables topographiques dans le plan, des deux premières composantes. 
rotation de $60^{\circ}$ environ, on obtient deux facteurs : $C^{\prime} 1$ est sensiblement l'altitude réelle $\mathrm{Z}$ du poste, $\mathrm{C}^{\prime} 2$, l'encaissement mesuré par TG1.

Dans le cas général, il paraît donc judicieux de caractériser la topographie par ces deux paramètres. Tous les autres en dérivent par combinaison linéaire.

Le cas des pluies joumalières décennales et des gradex d'hiver est peut-être un cas particulier. D'autres caractéristiques pluviométriques, pluies annuelles, pluies en une heure, peuvent très bien se projeter dans d'autres positions, comme c'est le cas pour le gradex d'été.

En essayant de relier la pluie à l'altitude réelle et à l'angle solide sous lequel on voit l'horizon, on est à peu près assuré de prendre en compte la plus grande partie de l'influence de la topographie sur la pluviométrie. $\mathrm{Ne}$ retenir, a priori, que l'altitude moyenne, présente donc un risque.

\section{Conclusions}

Elles portent sur deux aspects de ce travail : l'utilisation pratique des cartes, et les enseignements méthodologiques.

Sur le plan pratique, les cartes doivent être utilisées avec une prudence réfléchie. Les valeurs ponctuelles qui y figurent ne sont données qu'à $\pm 20 \%$ pour les gradex, et à $\pm 10 \%$ pour les pluies decennales. En ce qui concerne les isohyètes, leur tracé en fonction de la topographie peut être considéré comme le plus probable. Les incertitudes sont de l'ordre de $\pm 25 \%$ pour les gradex, et $\pm 13 \%$ pour les pluies decennales. La vocation de ce document est de permettre la détermination rapide des caractéristiques essentielles de la distribution statistique des pluies extrêmes sur la région. Il ne saurait cependant dispenser d'une étude plus approfondie dans chaque cas où la hauteur pluviométrique doit être évaluée avec précision.

Sur le plan méthodologique, les résultats sont très encourageants. Nous poursuivons l'amélioration de ces techniques, en élargissant le choix des variables pluviométriques à cartographier, (hauteurs de pluies interannuelles, fréquence des jours sans pluie ...) et des zones géographiques (France, Algérie, . . .). Certains inconvénients des méthodes décrites doivent disparaître avec l'introduction de régressions non linéaires, (mise en évidence d'un optimum pluviométrique par exemple), et de régressions variant selon la position dans le plan. Ces essais sont actuellement en cours. La difficulté est bien souvent, de trancher sur la signification statistique des résultats, au vu de l'imprécision des données de départ.

\section{Références}

[1] COLIN E., MEUNIER, PUECH - Problemes hydrologiques relatifs à l'étude des crues. Notes Techniques du C.T.G.R.E.F., Paris, 1972.

2] DEMASSIEUX L., LABORDE J.P., MARCHAND A. - Etude pluviométrique sur le District de l'Agglomération de Nancy. Connaître le sous-sol, un atout pour l'aménagement urbain. Actes du Colloque National, Lyon, 1979.

[3] ESTORGES J.L., LABORDE J.P., ZUMSTEIN J.F. - Mise en évidence des relations entre le gradex des pluies journalières et les gradex de durée inférieure à 24 heures en Lorraine. La Météorologie, numéro spécial Précipitations et $\mathrm{Hy}$ drologie, Paris, 1980.

[4] GUILLOTT P., DUBAND D. - La méthode du gradex pour le calcul de la probabilité des crues à partir des pluies, 1967. Les crues et leur évaluation. Actes du Colloque International A.I.H.S. Leningrad, 1967.

19

[5] LABORDE JP. - Notion d'indice de pente : approche par le calcul automatique. Sciences de la Terre, série informatique géologique, n 8 , Nancy, 1976

[6] LABORDE J.P. - Synthèse cartographique des pluies journalières extrémes dans l'Est de la France. S.R.A.E. Lorraine, D.E.D.S., Metz, 1980

[7] LABORDE J.P., SÁNCHEZ M. - Cartographie automatique des caractéristiques pluviométriques et exemple d'application dans l'Est de la France. Acte du Colloque International sur l'eau au XXIè siècle, Madrid, I980.

[8] MALLET J.L. - Présentation d'un ensemble de méthodes et techniques de la cartographie automatique. Sciences de la Terre, série informatique géologique, $n^{\circ} 4$, Nancy. 1974

[9] MALLET J.L. - Programmes de cartographie automatique : présentation de la bibliothèque CARTOLAB. Sciences de la Terre, série informatique géologique, $\mathrm{n}^{\circ} 7$, Nancy, 1976.

[10] SCHERER J.C. - Une méthode d'extrapolation dans l'espace de données pluviométriques moyennes. Recherche Géographique, $\mathrm{n}^{\circ} 4$, Strasbourg,

[11] Anonyme - Utilisation de quelques tests en hydrologie et calcul d'intervalles de confiance. Informations techniques du C.T.G.R.E.F., cahier $31, n^{\circ} 2$, Paris, 1978 .

[12] Anonyme - Techniques des barrages en aménagement rural. Ministère de l'Agriculture, Paris, 1977.

[13] Anonyme - Analyse des pluies de 1 à 10 jours. C.T.G.R.E.F., Antony, 1979.

[14] Anonyme - Synthese nationale sur les crues des petits bassins versants. Ministère de l'Agriculture, Paris, 1980.

[15] Anonyme - Hauteurs des précipitations journalières decennales de la France du Nord-Est. Carte au 1/500000 et sa Notice. Mission Déléguée de Bassin Rhin-Meuse. Metz, 1981. 